\title{
ON ABSTRACT DIFFERENTIAL EQUATIONS WITH NON INSTANTANEOUS IMPULSES
}

\author{
Eduardo Hernández - Michelle Pierri — Donal O’Regan
}

\begin{abstract}
We introduce a class of abstract differential equation with non instantaneous impulses which extend and generalize some recent models considered in the literature. We study the existence of mild and classical solution and present some applications involving partial differential equations with non-instantaneous impulses.
\end{abstract}

\section{Introduction}

In this work we introduce and study a new model of abstract impulsive differential equations which improve substantially the theory on differential equations with non-instantaneous impulsive introduced recently by Hernandez and O'Regan in [8]. Specifically, we study a class of abstract differential equations with non-instantaneous impulses of the form

$$
\begin{array}{rlrl}
u^{\prime}(t) & =A u(t)+f(t, u(t)), & t \in\left(s_{i}, t_{i+1}\right], i=0, \ldots, N, \\
u(t) & =h_{i}\left(t, u_{\left.\right|_{I_{i}(t)}}\right), & & t \in\left(t_{i}, s_{i}\right], i=1, \ldots, N, \\
u(0) & =x_{0}, & &
\end{array}
$$

where $A: D(A) \subset X \rightarrow X$ is the generator of a $C_{0}$-semigroup of bounded linear operators $(T(t))_{t \geq 0}$ defined on a Banach space $(X,\|\cdot\|), x_{0} \in X, 0=t_{0}=$ $s_{0}<t_{1} \leq s_{1} \leq t_{2}<\ldots t_{N} \leq s_{N} \leq t_{N+1}=a$ are pre-fixed numbers, the relation

2010 Mathematics Subject Classification. 34K30, 34K45, 35R12, 47D06.

Key words and phrases. Non-instantaneous impulses, impulsive differential equation, mild solution, partial differential equations with impulses. 
$t \rightarrow I_{i}(t)$ defines a $2^{[0, t]}$-set valued function, each function $h_{i}(t, \cdot)$ is a continuous function defined from a Banach space $\mathcal{C}_{i}(t)$ into $X$, the spaces $\mathcal{C}_{i}(t)$ are formed by function defined from $I_{i}(t)$ into $X$, the symbol $u_{\left.\right|_{I}}$ denotes the restriction of $u(\cdot)$ to an interval $I \subset[0, a]$ and $f:[0, a] \times X \rightarrow X$ is a suitable function.

To explain our motivations and objectives, we include some comments related to the problem studied in [8]. In Hernández and O'Regan [8] the authors introduced a new class of differential equations with impulses (called differential equation with non-instantaneous impulses) described in the form

$$
\begin{array}{rlrl}
u^{\prime}(t) & =A u(t)+f(t, u(t)), & t \in\left(s_{i}, t_{i+1}\right], i=0, \ldots, N, \\
u(t) & =g_{i}(t, u(t)), & & t \in\left(t_{i}, s_{i}\right], i=1, \ldots, N, \\
u(0) & =x_{0}, &
\end{array}
$$

where $A, f, x_{0}, s_{i}, t_{i}$ are as above and $g_{i} \in C\left(\left(t_{i}, s_{i}\right] \times X ; X\right)$ for all $i=1, \ldots, N$.

The main results in [8], see Theorems 2.1 and 2.2, are proved via fixed point techniques and assuming that the functions $g_{i}$ are globally Lipschitz. Specifically, the authors proved that the map $\Gamma: \mathcal{P C}(X) \rightarrow \mathcal{P C}(X)$ (see the definition of $\mathcal{P C}(X)$ below) given by $\Gamma u(0)=x_{0}, \Gamma u(t)=g_{i}(t, u(t))$ for $t \in\left(t_{i}, s_{i}\right]$ and

$$
\begin{array}{ll}
\Gamma u(t)=T\left(t-s_{i}\right) g_{i}\left(s_{i}, u\left(s_{i}\right)\right)+\int_{s_{i}}^{t} T(t-s) f(s, u(s)) d s, & t \in\left[s_{i}, t_{i+1}\right], \\
\Gamma u(t)=\int_{0}^{t} T(t-s) f(s, u(s)) d s, & t \in\left[0, t_{1}\right],
\end{array}
$$

have a fixed point $u \in \mathcal{P C}(X)$, which is called a mild solution of (1.4)-(1.5). A review of the proofs of the cited Theorems reveals that for each $i \in\{1, \ldots, N\}$, the map

$$
\Gamma_{i}: \mathcal{P C}(X)_{\mid\left(t_{i}, s_{i}\right]}=\left\{u \in C\left(\left(t_{i}, s_{i}\right] ; X\right): \lim _{t \downarrow t_{i}} u(t) \text { exists }\right\} \rightarrow \mathcal{P C}(X)_{\left.|| t_{i}, s_{i}\right]},
$$

given by $\Gamma_{i} u=g_{i}(\cdot, u(\cdot))_{\mid\left(t_{i}, s_{i}\right]}$ is a contraction on $\mathcal{P C}(X)_{\mid\left(t_{i}, s_{i}\right]}$ and there exists a unique function $v_{i} \in \mathcal{P C}(X)_{\left.\mid t_{i}, s_{i}\right]}$ such that $\Gamma_{i} v_{i}=v_{i}$. This can be a nonrealistic situation and it is quite restrictive for a general theory. However we note that the ideas and analysis in [8] partly motivates the general theory presented below.

Motivated by the above and by the fact that the above restriction arise from the abstract formulation of the problem (1.4)-(1.5), in this work we introduce a new abstract formulation for abstract differential equations with noninstantaneous impulses and we study the existence of mild and classical solutions for this type of problems.

Next we include some comments on the associated literature. The literature on abstract impulsive differential equations consider basically problems for which the impulses are abrupt and instantaneous. The literature on this type of problem is vast and different topics on the existence and qualitative properties 